Copyright (C 2012 IEEE. Personal use of this material is permitted. Permission from IEEE must be obtained for all other uses, in any current or future media, including reprinting/republishing this material for advertising or promotional purposes, creating new collective works, for resale or redistribution to servers or lists, or reuse of any copyrighted component of this work in other works. 


\title{
An Approach for Current Balancing in Distribution Networks with Rooftop PVs
}

\author{
Farhad Shahnia, Student Member, IEEE, Arindam Ghosh, Fellow, IEEE, \\ Gerard Ledwich, Senior Member, IEEE and Firuz Zare, Senior Member IEEE
}

\begin{abstract}
Current unbalance is a significant power quality problem in distribution networks. This problem increases further with the increased penetration of single-phase photovoltaic cells. In this paper, a new approach is developed for current unbalance reduction in medium voltage distribution networks. The method is based on utilization of three single-phase voltage source converters connected in delta configuration between the phases. Each converter is controlled to function as a varying capacitor. The combination of the load and the compensator will result in a balanced load with unity power factor. The efficacy of the proposed current unbalance reduction concept is verified through dynamic simulations in PSCAD/EMTDC.
\end{abstract}

Index Terms - Current Unbalance, Distribution Network, Voltage Source Converter.

\section{INTRODUCTION}

Current and voltage unbalance are one of the main power quality problems in Distribution Networks (DNs) [1]. The unbalance is more common in individual customer loads due to phase load inequality, especially where large single-phase loads are used. The electric utilities aim to distribute the residential loads equally among the three phases of distribution feeders [2]. However, although voltages are well balanced at the supply side, the voltages at the customer level can become unbalanced due to the unequal system impedances, unequal distribution of single-phase loads and unequal power consumption by houses or large number of single-phase transformers [2].

On the other hand, application of grid-connected Photovoltaic cells (PVs) is increasing in DNs around the world. Incentives by different countries promote the development of PVs connected to DNs. High penetration of intermittent, customer-owned and non-dispatchable PVs to the existing DNs can create different technical problems including current and voltage unbalance [3].

In the near future, it is expected the penetration level of Plug-in Electric vehicles (PEVs) will increase in DNs. PEVs can be viewed as large single-phase loads that will be added to the network and thus it can be expected that DN will have more problems on current and voltage unbalance [4].

This work was supported by Australian Research Council (ARC Discovery Grant DP 0774092).

F. Shahnia is with Department of Electrical and Computer Engineering, Curtin University, Perth, Australia. A. Ghosh, G. Ledwich and F. Zare are with School of Engineering Systems, Queensland University of Technology, Brisbane, Australia. (f.shahnia@qut.edu.au)
Current unbalance will result in voltage unbalance within the network. Voltage unbalance can result in overheating and de-rating of all induction motor types of loads [5]. It will also lead to mal-operation of protection relays and voltage regulation equipment in addition to generation of non-characteristic harmonics from power electronic loads [6].

The best solution is reducing the unbalance at the unbalance generation point. This can be easily achieved for industrial customers by installation of compensators. However, this is not very easy in residential DNs as there is not a single point of unbalance generation.

Different methods have been already studied and proposed for unbalance reduction in DNs. In [3], some conventional improvement methods such as feeder crosssection increase or capacitor installation are investigated for this purpose. However, these methods are expensive and not very efficient. In [7], the application of custom power devices including Distribution Static Compensator (DSTATCOM) and Dynamic Voltage Restorer (DVR) is proposed. It was proved that the custom power devices can fix their Point of Common Coupling (PCC) to a balanced voltage. Hence if the PCC voltage is balanced, the current drawn from the source (i.e. upper hand network) will be balanced. Hence, the unbalance will not be penetrated to upper hand level. In [8], the utilization of rooftop PVs for exchanging reactive power was proposed for balancing their PCC voltage. Although this method is very efficient in unbalance reduction, however it might take a few years for PV standards to be adopted for this strategy.

In this paper, a new method is proposed for unbalance reduction in a DN feeder. Three single-phase Voltage Source Converters (VSCs) are utilized on the DN feeder and controlled for current balancing at its PCC. By this method, the source current is kept balanced and hence the voltage unbalance is prevented from penetrating to upper hand network.

\section{CURRENT BALANCING THEORY}

Let us consider the power system structure shown in Fig. 1(a). It is assumed a three-phase balanced voltage source $\left(V_{A}, V_{B}\right.$ and $\left.V_{C}\right)$ is connected through feeder impedance $\left(Z_{F}\right)$ to the three unbalanced loads connected in delta $\left(Z_{A B}, Z_{B C}\right.$ and $\left.Z_{C A}\right)$. This will result in load current ( $\left.i_{\text {Load }}\right)$ and source current $\left(i_{S}\right)$ to be unbalanced.

Let us first ignore the feeder impedance. Now the PCC is directly connected to a stiff voltage source. Therefore, we can assume that 


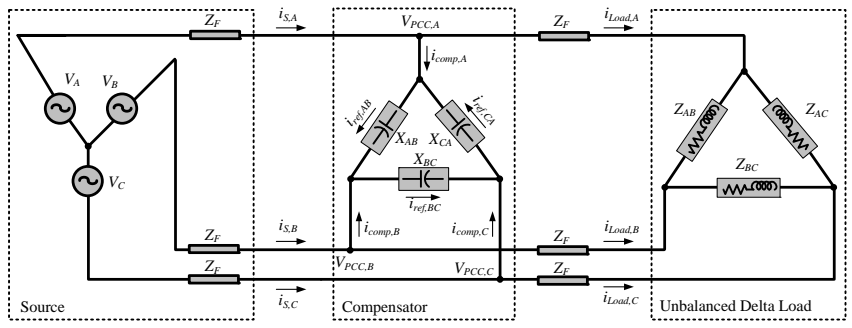

(a)

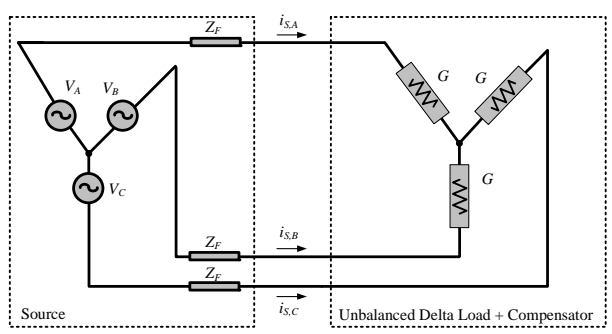

(b)

Fig. 1. Concept of the proposed current balancing method.

$$
\begin{aligned}
& V_{A}=V \angle 0 \quad V_{A B}=V_{A}-V_{B}=\sqrt{3} V \angle 30^{\circ} \\
& V_{B}=V \angle-120^{\circ} \quad \text { or } \quad V_{B C}=V_{B}-V_{C}=\sqrt{3} V \angle-90^{\circ} \\
& V_{C}=V \angle+120^{\circ} \quad V_{C A}=V_{C}-V_{A}=\sqrt{3} V \angle+150^{\circ}
\end{aligned}
$$

The source current can be made balanced if a compensator, composed of three unbalanced impedances $\left(X_{A B}, X_{B C}\right.$ and $X_{C A}$ ), is connected in parallel with the load in such a way that

$$
Z_{A B}\left\|X_{A B}=Z_{B C}\right\| X_{B C}=Z_{C A} \| X_{C A}
$$

Let the admittance of the delta connected load be given by

$$
Y_{A B}^{L}=\frac{1}{Z_{A B}}, \quad Y_{B C}^{L}=\frac{1}{Z_{B C}}, \quad Y_{C A}^{L}=\frac{1}{Z_{C A}}
$$

Then it has been in [9] that the sequence components of the load currents are then given by

$$
\begin{aligned}
& i_{\text {Load }, 0}=0 \\
& i_{\text {Load ,+ }}=\left(Y_{A B}^{L}+Y_{B C}^{L}+Y_{C A}^{L}\right) V \sqrt{3} \\
& i_{\text {Load ,- }}=-\left(a^{2} Y_{A B}^{L}+Y_{B C}^{L}+a Y_{C A}^{L}\right) V \sqrt{3}
\end{aligned}
$$

Let us define the susceptances of the compensator as

$B_{A B}^{\text {comp }}=\frac{1}{X_{A B}}, \quad B_{B C}^{\text {comp }}=\frac{1}{X_{B C}}, \quad B_{C A}^{\text {comp }}=\frac{1}{X_{C A}}$

Then, similar to (4), the symmetrical components of the compensator current are by

$$
\begin{aligned}
& i_{\text {comp }, 0}=0 \\
& i_{\text {comp },+}=j\left(B_{A B}^{\text {comp }}+B_{B C}^{\text {comp }}+B_{C A}^{\text {comp }}\right) V \sqrt{3} \\
& i_{\text {comp },-}=-j\left(a^{2} B_{A B}^{\text {comp }}+B_{B C}^{\text {comp }}+a B_{C A}^{\text {comp }}\right) V \sqrt{3}
\end{aligned}
$$

Since the zero-sequence is zero, the compensator has to cancel out the negative sequence completely, i.e., $i_{\text {comp ,- }}+i_{\text {Load ,- }}=0$

In addition, in order to have a unity power factor in the source currents, the imaginary part of the positive sequence needs to be cancelled as well

$\operatorname{Im}\left[i_{\text {comp },+}+i_{\text {Load },+}\right]=0$

Combining (7) and (8), the compensator susceptances can be calculated as

$$
\begin{aligned}
& B_{A B}^{\text {comp }}=-\frac{1}{3 V} \operatorname{Im}\left[i_{\text {Load }, A}+a \cdot i_{\text {Load }, B}-a^{2} \cdot i_{\text {Load }, C}\right] \\
& B_{B C}^{\text {comp }}=-\frac{1}{3 V} \operatorname{Im}\left[-i_{\text {Load }, A}+a \cdot i_{\text {Load }, B}+a^{2} \cdot i_{\text {Load }, C}\right] \\
& B_{C A}^{\text {comp }}=-\frac{1}{3 V} \operatorname{Im}\left[i_{\text {Load }, A}-a \cdot i_{\text {Load }, B}+a^{2} \cdot i_{\text {Load }, C}\right]
\end{aligned}
$$

Then, the load and compensator combination will look like a unity power factor load, as shown in Fig. 1(b).

Now, let us consider the following example.

Example 1: Let us assume a balanced three-phase source with a $11 \mathrm{kV}(\mathrm{L}-\mathrm{L}$, rms) is connected directly to a threephase delta load with unequal impedances of

$$
\begin{aligned}
& Z_{A B}=20+j 20 \Omega \\
& Z_{B C}=20 \Omega \\
& Z_{C A}=40+j 10 \Omega
\end{aligned}
$$

Hence the load currents are equal to

$$
\begin{aligned}
& i_{\text {Load }, A}=635.49 \angle-26.7^{\circ} \mathrm{A} \\
& i_{\text {Load }, B}=585.68 \angle-129.8^{\circ} \mathrm{A} \\
& i_{\text {Load }, C}=760.04 \angle+104.6^{\circ} \mathrm{A}
\end{aligned}
$$

From (9), the compensating susceptances are calculated as

$$
\begin{aligned}
& B_{A B}^{c o m p}=0.0097 \text { mho } \\
& B_{A B}^{c o m p}=0.00084904 \text { mho } \\
& B_{A B}^{c o m p}=0.0203 \text { mho }
\end{aligned}
$$

The connection of these susceptances in parallel with the load admittances will result in the following source currents

$$
\begin{aligned}
& i_{s, A}=625.74 \angle 0 \mathrm{~A} \\
& i_{s, B}=625.74 \angle-120^{\circ} \mathrm{A} \\
& i_{s, C}=625.74 \angle+120^{\circ} \mathrm{A}
\end{aligned}
$$

As can be seen, the source currents are now balanced and in phase with the source voltages.

Example 2: Let us now assume that feeder impedance is present. Therefore even if the phase-A source voltage has an angle of $0^{\circ}$, the angle of $V_{P C C, A}$ (see Fig. 1a) is not zero. Let us assume this angle to be $20^{\circ}$, i.e., the angle of $V_{P C C, A B}$ is $50^{\circ}$. The parallel combination of the susceptances given in Example-1 and the load admittances will result in the following source currents 


$$
\begin{aligned}
& i_{s, A}=628.08 \angle 0.34^{\circ} \mathrm{A} \\
& i_{s, B}=686.29 \angle-117.24^{\circ} \mathrm{A} \\
& i_{s, C}=682.91 \angle+117.37^{\circ} \mathrm{A}
\end{aligned}
$$

It can be seen that the theory fails here.

The reason for this failure is that the derivation of (9) is dependent on the L-L PCC voltage $V_{A B}$ to have a phase of $30^{\circ}$. Otherwise, the derivations presented in [9] are not valid. To overcome this problem, we first determine the phase of the PCC voltage $V_{A B}$. Let this be given by $\phi$. We know the computation is based on this being equal to $30^{\circ}$. We therefore define

$\delta=\phi-30^{\circ}$

The load and compensator currents then can be given by

$$
\begin{aligned}
& i_{\text {Load }, 0}=0 \\
& i_{\text {Load },+}=\left(Y_{A B}^{L}+Y_{B C}^{L}+Y_{C A}^{L}\right) V \sqrt{3} e^{j \delta} \\
& i_{\text {Load ,- }}=-\left(a^{2} Y_{A B}^{L}+Y_{B C}^{L}+a Y_{C A}^{L}\right) V \sqrt{3} e^{j \delta} \\
& i_{\text {comp }, 0}=0 \\
& i_{\text {comp ,+ }}=j\left(B_{A B}^{\text {comp }}+B_{B C}^{\text {comp }}+B_{C A}^{\text {comp }}\right) V \sqrt{3} e^{j \delta} \\
& i_{\text {comp ,- }}=-j\left(a^{2} B_{A B}^{\text {comp }}+B_{B C}^{\text {comp }}+a B_{C A}^{\text {comp }}\right) V \sqrt{3} e^{j \delta}
\end{aligned}
$$

From (11) and (12), it can be seen if the sequence components of the currents are multiplied by $e^{-j \delta}$, we can write these two equations in the same form as (4) and (6) respectively. Therefore the susceptances can be computed by modifying (9) as

$$
\begin{aligned}
& B_{A B}^{\text {comp }}=-\frac{1}{3 V} \operatorname{Im}\left\{\left[i_{\text {Load }, A}+a \cdot i_{\text {Load }, B}-a^{2} \cdot i_{\text {Load }, C}\right] e^{-j \delta}\right\} \\
& B_{B C}^{\text {comp }}=-\frac{1}{3 V} \operatorname{Im}\left\{\left[-i_{\text {Load }, A}+a \cdot i_{\text {Load }, B}+a^{2} \cdot i_{\text {Load }, C}\right] e^{-j \delta}\right\} \\
& B_{C A}^{\text {comp }}=-\frac{1}{3 V} \operatorname{Im}\left\{\left[i_{\text {Load }, A}-a \cdot i_{\text {Load }, B}+a^{2} \cdot i_{\text {Load }, C}\right] e^{-j \delta}\right\}
\end{aligned}
$$

Example 3: For the same value of phase angle given in Example-2, the susceptances are then computed to be the same as those given in Example-1. The computed source currents are then

$$
\begin{aligned}
& i_{s, A}=625.74 \angle 20^{\circ} \mathrm{A} \\
& i_{s, B}=625.74 \angle-100^{\circ} \mathrm{A} \\
& i_{s, C}=625.74 \angle+140^{\circ} \mathrm{A}
\end{aligned}
$$

It is expected that by adding the above mentioned compensator into the network, the source currents become balanced. In addition, the compensator will supply all the reactive power demand of the load and the PCC will look like a unity power factor. On the other hand, since the compensator only consists of susceptances, it will not consume any active power.

Since most of the distribution transformers have delta connection on their primary side (especially at medium voltage level), the load on a medium voltage feeder which supplies several distribution transformers can be assumed as an unbalanced three-phase delta connected load. Thus installation of the proposed compensator on a medium voltage distribution feeder can easily restrict and limit the current and voltage unbalance in these networks.

As mentioned in Section I, one of the main causes of current unbalance can be due to high penetration level of PVs. Let us assume that the generation capacity of the installed single-phase rooftop PVs on one phase is higher than the power demand on that phase. This will cause the excess of the power and current return back into the transformer in that phase. The resulting current unbalance can easily be removed by the proposed compensator.

\section{COMPENSATOR TOPOLOGY AND CONTROL}

Based on the current balancing theory mentioned in Section II, a compensator composed of three single-phase voltage source converters are utilized. Each VSC is composed of four IGBTs in parallel with reverse diodes. There is a single-phase transformer at the output of each converter. These three transformers are connected in $\mathrm{Y}-\Delta$ configuration. This is shown in Fig. 2.

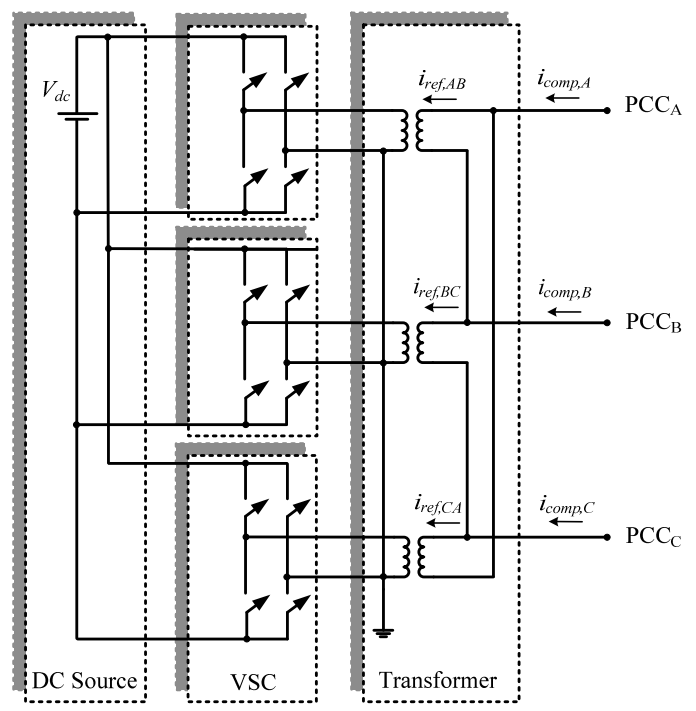

Fig. 2. The topology of the compensator utilized for current balancing.

By the delta connection of the secondary side of the transformers, each VSC is connected between two phases. Each VSC is to be controlled in such a way that it is seen as a susceptance equal to the values calculated in (13).

To implement the algorithm, first the positive sequence of the PCC voltage is calculated. From this, the angle $\delta$ is calculated. With this value of $\delta$, the susceptances are calculated using (13). Then the reference currents for the VSCs are calculated as

$$
\begin{aligned}
& i_{\text {ref }, A B}=j B_{A B}^{\text {comp }}\left(V_{P C C, A}-V_{P C C, B}\right) \\
& i_{r e f, B C}=j B_{B C}^{\text {comp }}\left(V_{P C C, B}-V_{P C C, C}\right) \\
& i_{\text {ref }, C A}=j B_{C A}^{\text {comp }}\left(V_{P C C, C}-V_{P C C, A}\right)
\end{aligned}
$$

Once the current references are obtained, they are tracked in hysteresis current control by each VSC by generating the appropriate firing angles of the IGBTs. 


\section{SIMULATION RESULTS}

For verifying the dynamic operation and characteristics of the proposed compensator, several cases are simulated in PSCAD/EMTDC, as described below.

\section{A. Case 1-Basic Principle}

The network of Fig. 1(a) is simulated in PSCAD/EMTDC with the technical data given in the Appendix. It is assumed at $t=0.5 \mathrm{~s}$, the compensator is connected to the network.

The instantaneous waveform of network source current is shown in Fig. 3(a) before and after the compensator connection. As it can be seen from this figure, the unbalanced source currents become balanced after compensator connection. The rms value of the source currents for each phase is shown in Fig. 3(b). It can be seen from this figure that all three phases have an equal current magnitude after the compensator connection.

The source current unbalance is shown in Fig. 3(C). The unbalanced is measured as the ratio of negative sequence current to the positive sequence current. This figure shows that the current unbalance is reduced from $38 \%$ to almost zero once the compensator is brought in.

Since the compensator uses a hysteresis current control, it can be expected that some harmonics are added to the network current and voltage. The Total Harmonic Distortion (THD) of the source current and PCC voltage is shown in Fig. 3(d). From this figure, it can be seen that the THD of voltage and current are both limited to $1 \%$.

The reactive power exchange of the source, compensator and the load is shown in Fig. 3(e). As expected, when the compensator is connected, it supplies all the reactive power demand of the load.

The PCC instantaneous voltage is shown in Fig. 3(f). Installation of the compensator should not have any effect on the PCC voltage as obvious from this figure.

In Fig. 3(f), voltage of phase- $A$ (scaled for comparison) and source current of phase- $A$ are shown. From this figure, it can be seen that in the presence of the compensator, the combination of the load and compensator draws current at unity power factor.

\section{B. Case 2- Dynamics}

The compensator must be capable of responding to the dynamics of the loads within the network. For verifying the dynamic operation and characteristics of the proposed compensator, another case is simulated. It is assumed that the compensator is connected at $t=0$. In addition, it is assumed that there is a load increase in the network at $t=$ $0.5 \mathrm{~s}$ and $t=1 \mathrm{~s}$ and a load decrease at $t=1.5 \mathrm{~s}$. The power in each phase of the network changes as shown in Fig. 4(a). The rms values of the load current are similar to the power in each phase. The rms values of the source current for all three phases are as shown in Fig. 4(b). This proves the dynamic compatibility of the proposed compensator. This is achieved as the susceptance values of the compensator, calculated from (11), vary based on the load current as shown in Fig. 4(c). (a)
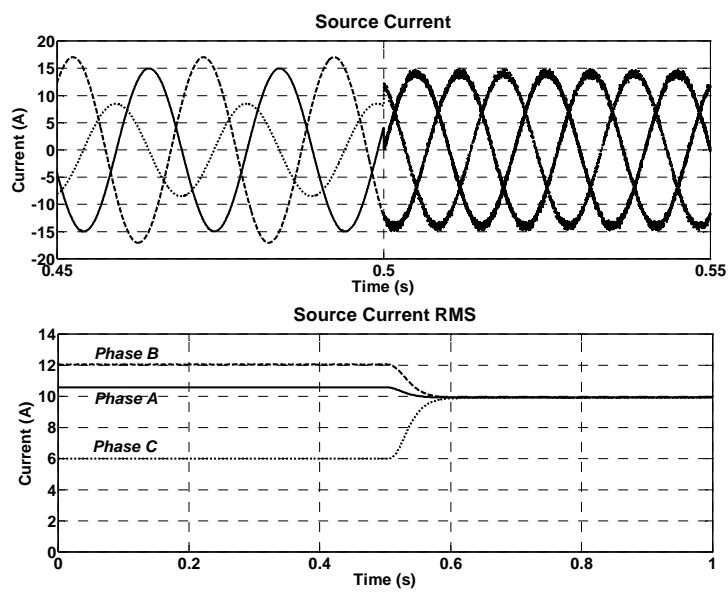

Source Current Unbalance

(c)
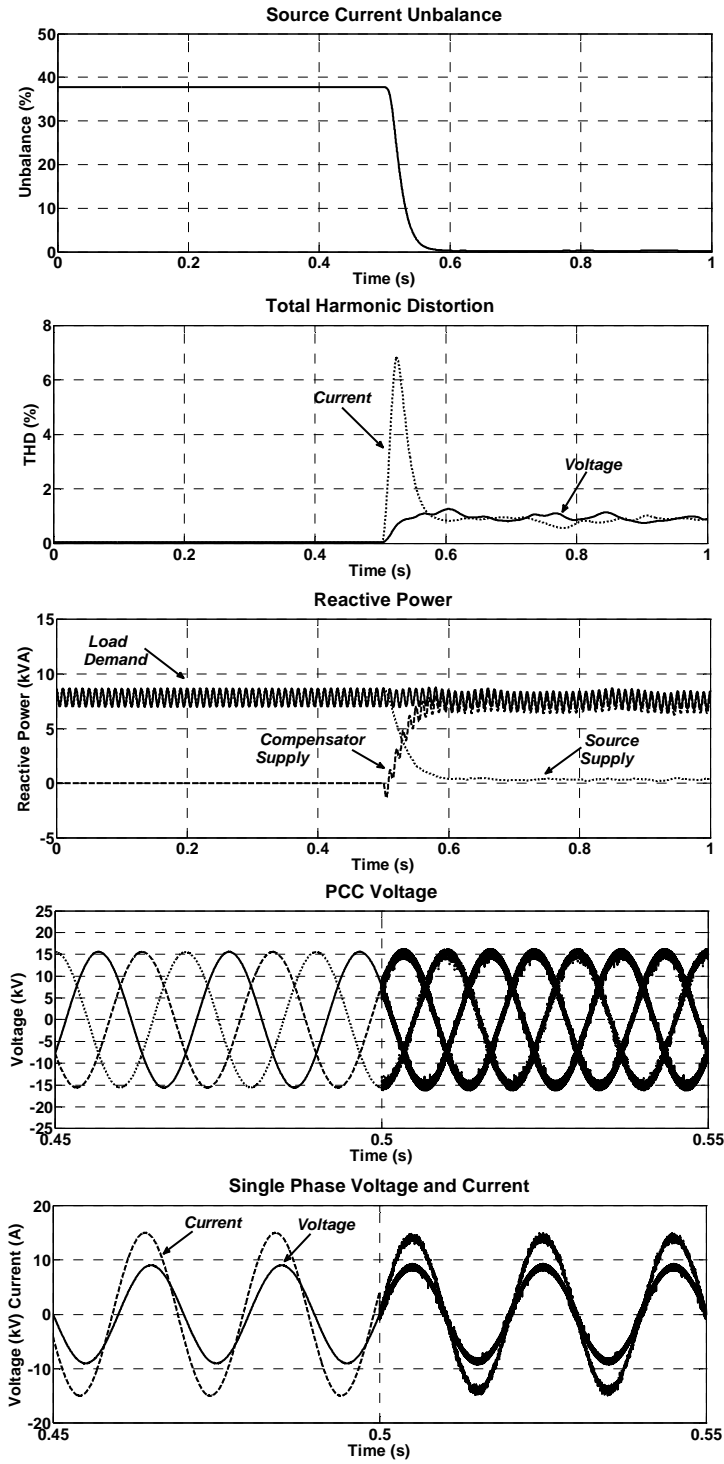

Fig. 3. Simulation results for Case-1.

\section{Case 3- Low Voltage Distribution Network}

As explained in Section II, most of the distribution transformers have delta connection on their primary side. Thus the compensator installed on a medium voltage (e.g. $11 \mathrm{kV}$ ) which supplies several distribution transformers, can 
restrict the current unbalance in the medium voltage levels. This is a main functionality for this compensator. For verifying this, another case is studied as shown in Fig. 5. It is assumed that a distribution transformer is feeding a threephase unbalanced star-connected load. In addition to that, for studying a very worst case, a single-phase rooftop PV is connected to one phase. This causes a reverse current flow (i.e. 180 degree phase shift) in that phase. It is assumed that the compensator is connected at $t=0.5 \mathrm{~s}$. The three-phase source current is shown before and after compensator in Fig. 6 . This proves the efficacy of the proposed compensator.

(a)
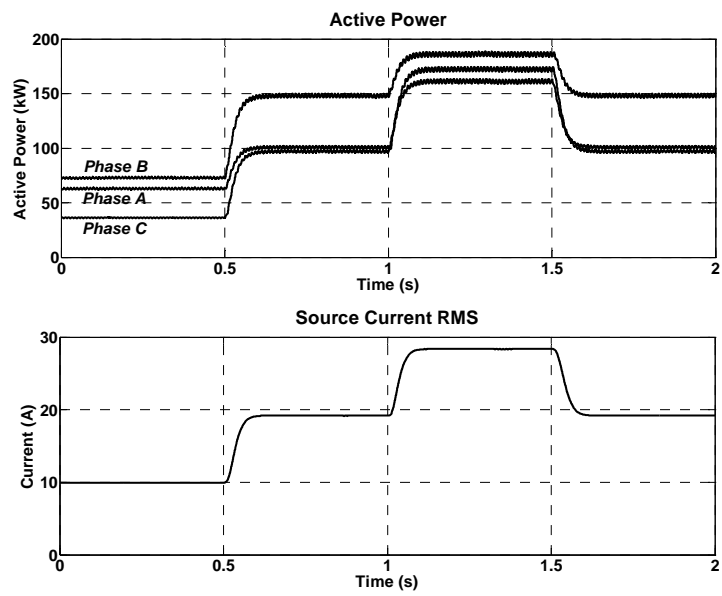

(b)

Susceptance

(c)

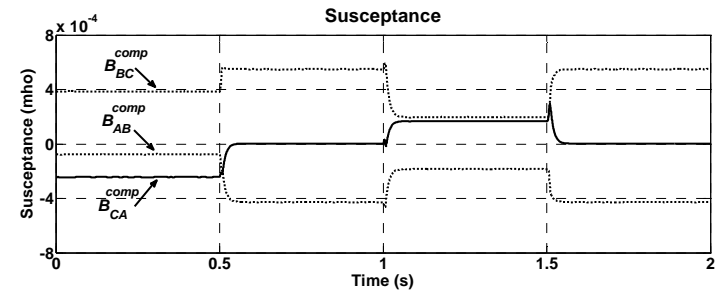

Fig. 4. Simulation results for Case-2.

\section{Case 4- Multiple Installations along the Feeder}

The proposed topology has the capability of being installed at multiple locations along a feeder. This is very useful in very long feeders to prevent to restrict the current unbalance at different distances. Another case study is carried out assuming the proposed compensator is installed at 2 different locations along the feeder. Compensator- 1 is installed at the beginning of the feeder and compensator- 2 at the middle of the feeder, as shown in Fig. 7. For studying the effect of each compensator, it is assumed that the compensator- 2 connects at $t=0.5 \mathrm{~s}$ and compensator- 1 at $t=1 \mathrm{~s}$. The current rms for the upper hand side of each compensator is as shown in Fig. 8. From this figure, it is shown that each compensator is capable of effectively balancing its source current without any conflicts with each other. In addition, in this way, each compensator is responsible for generating the reactive power demand of the loads in its downstream level. Thus, this will reduce the rating of the compensator if multiple ones are used instead of a single compensator at the beginning of the feeder.

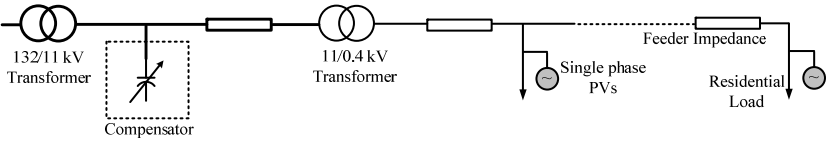

Fig. 5. Network under consideration for Case-3.

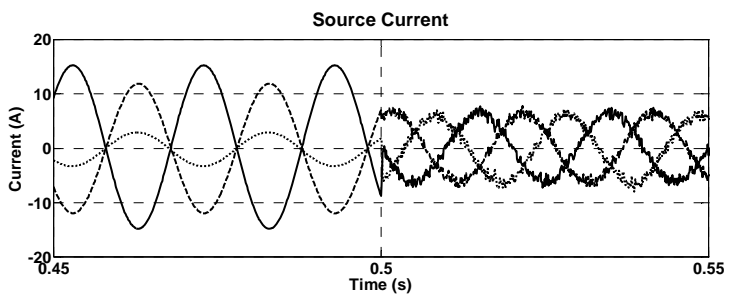

Fig. 6. Simulation results for Case-3.

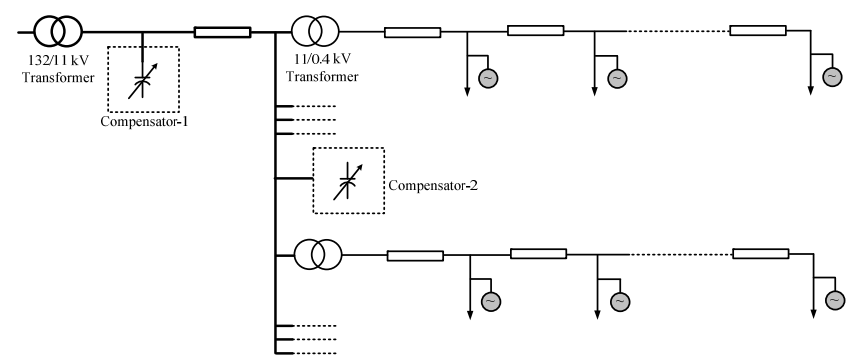

Fig. 7. Network under consideration for Case-4.

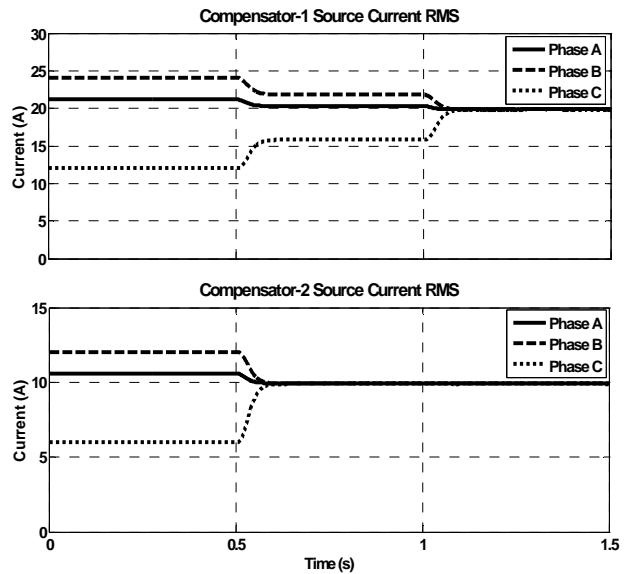

Fig. 8. Simulation results for Case-4.

\section{CONCLUSIONS}

Current unbalance is a significant power quality problem in Distribution Networks. Penetration of photovoltaic cells and plug-in electric vehicles will result in higher current unbalance in the network. A new approach was developed in this paper for current unbalance reduction in medium voltage distribution networks. The proposed method utilized three single-phase voltage source converters, connected in delta configuration between the phases, to function as a varying capacitor between the phases. In this way, the combination of the load and the proposed compensator seemed as a balanced load with unity power factor. The dynamic behavior and efficacy of the proposed concept was verified through simulations in PSCAD/EMTDC. 
APPENDIX

Table 1 Technical Parameters of the case studies

$\begin{array}{ll}\text { Source Voltage } & 11 \mathrm{kV}(\mathrm{L}-\mathrm{L} \mathrm{rms}) \\ \text { Feeder Impedance } & 50 \mathrm{~mm}^{2} \mathrm{ACSR},(\mathrm{Z}=1.08+\mathrm{j} \times 0.302) \Omega \\ \text { Delta Loads } & Z_{A B}=1200.2+j 62.8 \\ & Z_{B C}=2400.2+j 94.2 \\ & Z_{C A}=1200.2+j 62.8 \\ \text { LV Transformer } & 11 / 0.415 \mathrm{kV}, 50 \mathrm{~Hz}, 100 \mathrm{kVA}, \Delta-\mathrm{Y}_{\mathrm{G}}, Z_{I}=5 \% \\ \text { LV Network Star Load } & Z_{A}=0.482+j 6.28 \\ & Z_{B}=0.241+j 9.42 \\ & Z_{C}=0.120+j 6.28\end{array}$

\section{REFERENCES}

[1] A. Ghosh and G. Ledwich, Power Quality Enhancement Using Custom Power Devices, Kluwer Academic Publishers, 2002.

[2] T. A. Short, Electric Power Distribution Handbook: CRC Press, 2004.

[3] F. Shahnia, R. Majumder, A. Ghosh, G. Ledwich and F. Zare, "Voltage unbalance analysis in residential low voltage distribution networks with rooftop PVs,” Electric Power Systems Research, Volume 81, Issue 9, September 2011, Pages 1805-1814.

[4] F. Shahnia, A. Ghosh, G. Ledwich and F. Zare, "Voltage unbalance sensitivity analysis of plug-in electric vehicles in distribution networks," in Proc. of Australian Universities Power Engineering Conf. (AUPEC), Sep. 2011, Australia.

[5] P. Gnacinski, "Windings Temperature and Loss of Life of an Induction Machine Under Voltage Unbalance Combined With Over- or Undervoltages,” IEEE Trans. on Energy Conversion, vol. 23, pp. 363371, 2008.

[6] A. von Jouanne and B. Banerjee, "Assessment of voltage unbalance," IEEE Trans. on Power Delivery,, vol. 16, pp. 782-790, 2001.

[7] F. Shahnia, A. Ghosh, G. Ledwich and F. Zare, "Voltage correction in low voltage distribution networks with rooftop PVs using custom power devices," in Proc. of $37^{\text {th }}$ Annual Conf. of IEEE Industrial Electronics Society, Nov. 2011, Australia.

[8] F. Shahnia, Analysis and Correction of Voltage Profile in Low Voltage Distribution Networks Containing Photovoltaic Cells and Electric Vehicles, PhD Thesis, Queensland University of Technology, Australia, 2011.

[9] T.J. Miller, Reactive Power Control in Electric Systems, Wiley, 1982.
Farhad Shahnia received his B.Sc. and M.Sc. in Electrical Power Engineering with first class honor from University of Tabriz, Tabriz, Iran in 2004 and 2006, respectively and his Ph.D. Electrical Engineering from Queensland University of Technology, Brisbane, Australia in 2011. His is currently a research fellow in Queensland University of Technology and will soon move to Curtin University in Perth, Australia. His fields of interest include Distribution Networks, Power Quality, DG and Power Electronics application in Power Systems.

Arindam Ghosh (S'80, M'83, SM'93, F'06) is the Professor of Power Engineering at Queensland University of Technology, Brisbane, Australia. He has obtained a Ph.D. in EE from University of Calgary, Canada in 1983. Prior to joining the QUT in 2006, he was with the Department of Electrical Engineering at IIT Kanpur, India for 21 years. He is a Fellow of Indian National Academy of Engineering and IEEE. His interests are in Control of Power Systems and Power Electronic Devices.

Gerard Ledwich (M'73, SM'92) received the Ph.D. in electrical engineering from the University of Newcastle, Australia, in 1976. He has been Chair Professor in Power Engineering at Queensland University of Technology, Australia since 2006. Previously he was the Chair in Electrical Asset Management from 1998 to 2005 at the same university. He was Head of Electrical Engineering at the University of Newcastle from 1997 t0 1998. Previously he was associated with the University of Queensland from 1976 to 1994 . His interests are in the areas of power systems, power electronics and controls. He is a Fellow of I.E.Aust.

Firuz Zare (M’97, SM’06) was born in Iran in 1967. He holds a PhD degree in Electrical Engineering from Queensland University of Technology in Australia. He has worked as a development engineer and a consultant in industry for several years. He has joined the school of engineering systems in QUT in 2006. His research interests are power electronic applications, pulse-width modulation techniques, renewable energy systems and electromagnetic interferences. 